ENTREPRENEURSHIP AND SUSTAINABILITY ISSUES

ISSN 2345-0282 (online) http://jssidoi.org/jesi/ 2020 Volume 8 Number 1 (September)

http://doi.org/10.9770/jesi.2020.8.1(30)

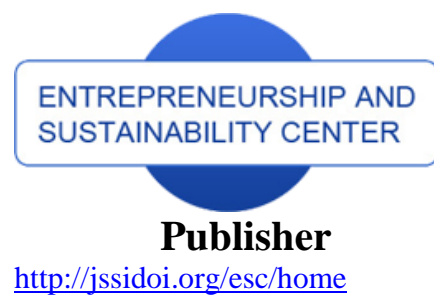

enterprise

europe

network

$x_{*} *_{*}^{*}$

Business Support on Your Doorstep

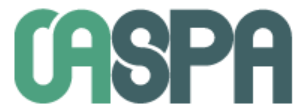

Scopus

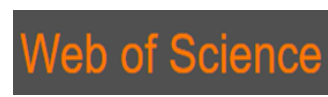

http://jssidoi.org/esc/home

\title{
THE INFLUENCE OF THE CLASSIFICATION OF NON-CURRENT ASSETS AS HELD FOR SALES ON THE LIQUIDITY OF THE COMPANY'S BALANCE SHEET
}

\author{
Mariya M. Shygun ${ }^{1}$, Nataliia A. Ostapiuk ${ }^{2}$, Oksana V. Zayachkivska ${ }^{3}$, Natalia V. Goylo ${ }^{4}$ \\ ${ }^{1,2}$ Accounting and Taxation Department, Kyiv National Economic University named after Vadym Hetman, 03057, Kyiv, \\ Ukraine \\ ${ }^{3}$ Department of Finance and Environmental Economics, National University of Water Management and Environmental \\ Management, 33000, Rivne, Ukraine, \\ ${ }^{4}$ Department of Audit, Kyiv National Economic University named after Vadym Hetman, 03057, Kyiv, Ukraine \\ E-mails: ${ }^{1}$ shygun@ukr.net, ${ }^{2}$ ostapiuk@ukr.net , ${ }^{3}$ o.v.zaychkivska@gmail.com, ${ }^{4}$ goylo@ukr.net
}

Received 10 June 2020; accepted 25 June 2020; published 30 September 2020

\begin{abstract}
Enterprises are in constant search for additional sources of financing their activities and increasing the liquidity of their balance. One of these methods is the classification of non-current assets as held for sale, which causes changes in the structure of assets of the company, affects its liquidity and solvency. At the same time, users of information should be sure of the reality of such operations for the classification of non-current assets. It is important to avoid manipulating balance sheets and artificially enhancing their liquidity by changing the structure of assets. The results of a study of the impact of operations on classification of non-current assets as held for sale on the liquidity of the company's balance sheet are presented in this article. The impact of non-current assets held for sale on liquidity is shown separately provided that they are included in the groups of fast-selling assets and slow-selling assets. The impact of such groupings on the liquidity indicators is shown by means of coefficient analysis. The nature and level of the impact of non-current assets held for sale on the liquidity of the balance sheet is identified by modelling a consistent change in their volume relative to the value of non-current assets and capital of the company. For the purpose of effective asset management, the optimal indicator of non-current assets held for sale in the company's balance sheet has been proposed, which avoids the risk of lowering the productivity of the existing equipment while ensuring the release of financial resources due to the sale of assets.
\end{abstract}

Keywords: non-current assets; held for sale; liquidity; liquidity analysis; ratio analysis of liquidity; capital; reclassification of non-current assets

Reference to this paper should be made as follows: Shygun, M.M., Ostapiuk, N.A., Zayachkivska, O.V., Goylo, N.V. 2020. The influence of the classification of non-current assets as holding for sales on the liquidity of the company's balance sheet. Entrepreneurship and Sustainability Issues, 8(1), 430-441. http://doi.org/10.9770/jesi.2020.8.1(30)

JEL Classifications: M41, M21, G32

Additional disciplines: General Statistical Simulation Methods 


\section{ENTREPRENEURSHIP AND SUSTAINABILITY ISSUES}

ISSN 2345-0282 (online) http://jssidoi.org/jesi/

2020 Volume 8 Number 1 (September)

http://doi.org/10.9770/jesi.2020.8.1(30)

\section{Introduction}

The activity of modern economic entities is accompanied by a high degree of risk and variability of the environment. Enterprises feel the constant influence of risk factors on their financial position, liquidity and solvency. One way to increase liquidity and find additional sources of financing is to transfer assets from noncurrent to current assets for resale. Such object as non-current assets held for sale (hereinafter - NAHS), is formed as a result of these classification procedures.

In economically developed countries, non-current assets held for sale occupy a share in the assets for $0.1 \%$ to 6.4\% (according to the financial statements of the oil and gas companies of the United Kingdom, France, USA, Poland for 2007-2018). NAHS are an effective management tool for structure of assets of an entity, they help in regulation of its level of liquidity and solvency, as well as represent a source of investments in the material base of activity.

The need for effective management of such kind assets as non-current assets held for sale requires the development of appropriate analytical procedures for the purpose of determining the indicators of their condition and dynamics, and conducting ratio analysis. It should be noted that at present there are no ready-made decisions and special studies on the analysis of transactions with non-current assets held for sale.

\section{The purpose and methodology of the research}

The purpose of this article is to investigate the impact of the classification of non-current assets as held for sale on the company's balance sheet liquidity, and to substantiate the asset groups to which non-current assets held for sale should be included in conducting the liquidity analysis.

The analytical methods based on the construction of qualitative groupings for the creation of key groups of assets and liabilities, are the basis of the presented study. Identifying structural and temporal changes in the company's assets and liabilities by major groups of them is carried out by determining the indicators of structure and dynamics. The ratio analysis allowed us to calculate liquidity ratios, followed by a systematic approach to finding solutions and recommendations for users of liquidity analysis results. Methods of analysis and synthesis, induction and deduction, structural and logical methods, methods of idealization and generalization were used in the construction of theoretical foundations of the presented research, the identification of problematic issues, the formulation of hypotheses and the formation of conclusions.

\section{Results of the research}

Problems of the analysis of non-current assets are of high scientific interest of scientists and researchers. In particular, the authors investigate approaches to the analysis of the structure of property of the company (Gabrusewicz 2018), evaluation of the effectiveness of the use of non-current assets (Khalatur, 2016), analysis of impairment of non-current assets (Nawaiseh 2015), analysis of the structure and use of non-current assets (Kalubi 2017; Kulko-Labyntseva 2014; Paliukh, Matviychuk 2015; Selivanov 2016), the analysis of the sale of noncurrent assets (Cieśla 2016; Kraszewska-Szuba 2017; Lazarowicz 2018), the analysis of the availability, condition and efficiency of the use of intangible assets (Poliova, Dubovets 2010; Rozeliuk, Denchuk 2006; Cherep, Pukhalska 2011; Chub 2014) etc.

The analysis of liquidity of economic entities is widely disclosed in the research of scientists, among them approaches to classification of assets by the degree of liquidity (Ivashevska 2006), effects of changes in balance 


\section{ENTREPRENEURSHIP AND SUSTAINABILITY ISSUES}

ISSN 2345-0282 (online) http://jssidoi.org/jesi/ 2020 Volume 8 Number 1 (September) http://doi.org/10.9770/jesi.2020.8.1(30)

sheet on liquidity ratios (Stupnytska 2014), the system of indicators of liquidity analysis (Teren 2016), the study of the balance sheet liquidity (Tiuleneva 2013) and others.

However, the analysis of transactions with non-current assets held for sale and their impact on the balance sheet liquidity still scarcely explored. In addition, such type of analysis has a number of unresolved issues, in particular, to which group of assets should non-current assets held for sale be classified in the analysis of liquidity, as fastselling or slow-selling. Also a problematic issue is determining the optimum indicator of NAHS in the company's balance sheet, that would allow to avoid the risk of diminishing the productivity of the existing equipment, and thus contribute to the release of financial resources resulting from the sale of assets.

In international practice, the accounting for non-current assets held for sale is governed by IFRS 5 "Non-current Assets Held for Sale and Discontinued Operations". It is necessary to take into account the conditions of recognition set out in paragraphs 6-9 of IFRS 5 in order to classify assets as a group of non-current assets held for sale:

1) the reimbursement of the carrying amount of an asset occurs as a result of a sale transaction;

2) the asset must be in a condition suitable for immediate sale;

3) the asset must be fit for sale on terms that are common to such assets;

4) the high probability of sale of such assets is ensured and the procedures, promotion of the asset in the market at current fair value (sales plan, buyer determination program) are performed;

5) the sale will take place within one year from the date of classification (except for the effects of events independent of the entity and the cases specified in paragraph 9 of IFRS 5).

The reasons for reclassifying non-current assets and selling them may be different, and may include planned activities to upgrade the material base of production (the sale of worn-out equipment requires constant repairs, requires refurbishment, re-equipment, does not provide the required production capacity, etc.) as well as forced search for free financial resources (sale of available components of non-current assets for release of cash to cover urgent liabilities).

The company receives significant benefits from the reclassification of non-current assets, regardless of the reasons for such operations (Table 1).

Table 1. Advantages for a company from classifying non-current assets as held for sale

\begin{tabular}{|c|c|}
\hline \multicolumn{2}{|c|}{ Advantages } \\
\hline Material content & Financial content \\
\hline $\begin{array}{l}\text { reduces or eliminates equipment that has moderate and / or high } \\
\text { levels of physical or moral obsolescence }\end{array}$ & creates a reserve to increase funding for activities \\
\hline $\begin{array}{l}\text { eliminates equipment that not used in operating activities (due to } \\
\text { falling demand for products, occurrence of substitute products, loss } \\
\text { of market share, etc.) }\end{array}$ & $\begin{array}{l}\text { is a source of growth in the solvency of a company as a result of } \\
\text { the release of additional financial resources }\end{array}$ \\
\hline $\begin{array}{l}\text { reduces or eliminates equipment that requires high maintenance, } \\
\text { repair or retrofitting }\end{array}$ & $\begin{array}{l}\text { is a tool to increase the liquidity of assets by managing their } \\
\text { structure }\end{array}$ \\
\hline $\begin{array}{l}\text { reduces the volume or eliminates equipment that needs urgent } \\
\text { upgrading as a result of reduced productivity, the emergence of } \\
\text { technologically new types of production }\end{array}$ & is a source of investment in fixed assets and their updating \\
\hline
\end{tabular}

Source: The authors

Considering the significant benefits of classifying non-current assets as held for sale, companies may use artificial manipulation of financial statements to enhance balance sheet liquidity in individual cases. The risk of 


\section{ENTREPRENEURSHIP AND SUSTAINABILITY ISSUES}

ISSN 2345-0282 (online) http://jssidoi.org/jesi/

2020 Volume 8 Number 1 (September)

http://doi.org/10.9770/jesi.2020.8.1(30)

manipulation requires increasing attention of analysts and other users to the financial statements, which presents the non-current assets held for sale, as well as the reliability of their valuation and validity of recognition.

Conducting liquidity analysis of the balance sheet involves execution of standard procedures for grouping assets and liabilities. In particular, assets are divided into groups according to their degree of liquidity, and liabilities for the urgency maturity: A1 - absolutely liquid and highly liquid assets; A2 - fast-selling assets; A3 - slowly realized assets; A4 - hard-to-sell assets; L1 is the most urgent liabilities; L2 - current liabilities; L3 - long-term and other long-term liabilities; L4 - permanent liabilities. A more detailed division of assets and liabilities into 6 groups may be applied respectively in some cases.

The company's balance sheet will be considered as liquid if its current assets will exceed or match the volume of its current liabilities. The following correspondences between asset and liability groups should also be maintained in order to ensure the liquidity:

\begin{tabular}{|c|c|c|c|c|}
\hline & Asset groups & Correlation & & Liability groups \\
\hline A1 & $\begin{array}{l}\text { absolutely liquid and highly } \\
\text { liquid assets }\end{array}$ & $\geq$ & L1 & the most urgent liabilities \\
\hline A2 & fast-selling assets & $\geq$ & L2 & current liabilities \\
\hline A3 & slowly realized assets & $\geq$ & L3 & $\begin{array}{l}\text { long-term and other long-term } \\
\text { liabilities }\end{array}$ \\
\hline A4 & hard-to-sell assets & $\leq$ & $\mathrm{L} 4$ & permanent liabilities \\
\hline
\end{tabular}

Performing classification of non-current assets as held for sale requires changing the structure of the asset groups according to their liquidity level. Thereafter, non-current assets from Group A4 of hard-to-sell illiquid assets are transferred to Group A3 of slowly realized assets as they are highly likely to be sold within one year from the date of reclassification. At the same time, they can also be transferred to group A2 of fast-selling assets if their sale date is clearly defined within the next few months. The choice of group A2 or A3 should be clearly substantiated by the appropriate grounds in conducting a liquidity analysis.

It is important for stakeholders to have accurate information about the composition and structure of a company's assets, as inaccurate data may distort the results of the company's financial position analysis, its liquidity and solvency. The negative impact of the decisions made on the basis of unreliable data may be the provision of credit or investment resources to the company, which will not be able to cover its commitments in the future.

The criteria for assigning non-current assets after their reclassification in NAHS to group A2 of fast-selling assets or A3 of slowly realized assets for the purposes of liquidity analysis can be obtained on the basis of comparative study of the two mentioned approaches to grouping on the example of a real entity.

The calculations presented in this article are based on the financial statements of PKN ORLEN (Poland) for 20072018. The performed structuring of assets by liquidity level and liabilities by maturity is presented in Table 2 .

Given actual data of PKN ORLEN reflects the volumes of the respective asset groups in terms of their liquidity [A1-A4] and liabilities for their maturity [L1-L4], according to which NAHS compile $0.06-0.7 \%$ of the value of non-current assets of the company and $0.03-0.41 \%$ of its capital. 


\section{ENTREPRENEURSHIP AND SUSTAINABILITY ISSUES}

ISSN 2345-0282 (online) http://jssidoi.org/jesi/ 2020 Volume 8 Number 1 (September) http://doi.org/10.9770/jesi.2020.8.1(30)

Table 2. Asset and Liability Grouping of PKN ORLEN (Poland) for 2007-2018 for the purposes of liquidity analysis (PLN million)

\begin{tabular}{|c|c|c|c|c|c|c|c|c|c|c|c|c|c|c|c|c|c|c|c|c|c|c|c|c|}
\hline \multirow{2}{*}{$\begin{array}{l}\text { Groups of } \\
\text { assets, } \\
\text { liabilities }\end{array}$} & \multicolumn{2}{|c|}{2007} & \multicolumn{2}{|c|}{2008} & \multicolumn{2}{|c|}{2009} & \multicolumn{2}{|l|}{2010} & \multicolumn{2}{|c|}{2011} & \multicolumn{2}{|c|}{2012} & \multicolumn{2}{|c|}{2013} & \multicolumn{2}{|c|}{2014} & \multicolumn{2}{|c|}{2015} & \multicolumn{2}{|c|}{2016} & \multicolumn{2}{|c|}{2017} & \multicolumn{2}{|c|}{2018} \\
\hline & Value & $\%$ & Value & $\%$ & Value & $\%$ & Value & $\%$ & Value & $\%$ & Value & $\%$ & Value & $\%$ & Value & $\%$ & Value & $\%$ & Value & $\%$ & Value & $\%$ & Value & $\%$ \\
\hline 1 & 2 & 3 & 4 & 5 & 6 & 7 & 8 & 9 & 10 & 11 & 12 & 13 & 14 & 15 & & 17 & & 19 & 20 & 21 & 22 & 23 & 54 & 25 \\
\hline \multicolumn{25}{|c|}{ NAHS as a part of hard-to-sell assets (A4) } \\
\hline A1 & 1666,2 & 3,6 & 1602,1 & 3,4 & 3122,1 & 6,4 & 3045,3 & 6,0 & 5702,6 & 9,7 & 2579,6 & 4,9 & 2854,0 & 5,56 & \begin{tabular}{|l|}
4799,0 \\
\end{tabular} & 10,3 & \begin{tabular}{|l|}
3154,0 \\
\end{tabular} & 6,6 & 5169,0 & 9,3 & 6678,0 & 11,0 & 4716,0 & \begin{tabular}{|l|}
7,4 \\
\end{tabular} \\
\hline A2 & 10884,3 & 23,6 & 10366,9 & 22,1 & 9698,9 & 19,8 & \begin{tabular}{|l|}
11091,7 \\
\end{tabular} & 21,7 & 714075,4 & 24,01 & 14533,7 & 27,6 & 14388,0 & 28,0 & 11870,0 & 25,4 & 10604,0 & 22,01 & 12335,0 & 22,2 & 13838,0 & 22,8 & 10593,0 & 16,5 \\
\hline A3 & 6627,9 & 14,4 & \begin{tabular}{|l|}
5674,5 \\
\end{tabular} & 12,1 & 6590,0 & 13,4 & \begin{tabular}{|l|}
6540,2 \\
\end{tabular} & 12,8 & 10325,8 & 17,6 & 8642,3 & 16,4 & \begin{tabular}{l|l|}
7188,0 \\
\end{tabular} & 14,0 & \begin{tabular}{|l|}
5051,0 \\
\end{tabular} & 10,8 & \begin{tabular}{|l|}
6920,0 \\
\end{tabular} & 14,4 & 7673,0 & 13,8 & 8333,0 & 13,7 & 14666,8 & 22,9 \\
\hline A4 & 26924,5 & 58,4 & 29332,4 & 62,4 & 29687,2 & 60,5 & \begin{tabular}{|l|}
30472,5 \\
\end{tabular} & 59,6 & 28627,7 & 48,72 & 26875,3 & 51,1 & 26922,0 & 52,4 & 25005,0 & 53,5 & \begin{tabular}{|l|}
27459,0 \\
\end{tabular} & 57,03 & 30382,0 & 54,7 & 31815,0 & 52,4 & 34055,2 & 53,2 \\
\hline Capital & 46102,8 & 100,0 & \begin{tabular}{|l|l}
46975,7 & 1 \\
\end{tabular} & 100,0 & 49088,11 & 100,0 & \begin{tabular}{|l|l|}
51149,81 \\
\end{tabular} & 100,0 & 58731,5 & 100,05 & 52630,8 & 100,0 & 51352,0 & 100,0 & \begin{tabular}{|l|l}
46725,0 & 1 \\
\end{tabular} & 100,0 & 48137,0 & 100,05 & 55559,0 & 100,0 & 60664,0 & 100,0 & 64031,0 & 100,0 \\
\hline L1 & 39,4 & 0,1 & \begin{tabular}{|l|}
35,5 \\
\end{tabular} & 0,1 & 24,2 & 0,1 & \begin{tabular}{|r|}
23,4 \\
\end{tabular} & 0,1 & 673,6 & 1,15 & 83,7 & 0,2 & 36,0 & 0,07 & \begin{tabular}{|l|}
42,0 \\
\end{tabular} & 0,1 & 162,0 & \begin{tabular}{|l|}
0,3 \\
\end{tabular} & 659,0 & 1,2 & 290,0 & 0,5 & 473,0 & 0,7 \\
\hline $\mathrm{L} 2$ & 9896,2 & 21,5 & 9626,8 & 20,5 & 11612,0 & 23,7 & \begin{tabular}{|c|}
13580,5 \\
\end{tabular} & 26,6 & 15534,8 & 26,51 & 12777,9 & 24,3 & 14123,0 & 27,5 & 12235,0 & 26,2 & 11728,0 & 24,41 & 14308,0 & 25,8 & 15102,0 & 24,9 & 14213,0 & 22,2 \\
\hline & 2503,1 & 5,4 & 12147,7 & 25,9 & \begin{tabular}{|l|}
2521,3 \\
\end{tabular} & 5,1 & \begin{tabular}{|l|}
2621,1 \\
\end{tabular} & 5,1 & \begin{tabular}{|l|}
3604,3 \\
\end{tabular} & 6,1 & 2265,7 & 4,3 & \begin{tabular}{|l|l|}
1795,0 \\
\end{tabular} & 3,5 & \begin{tabular}{|l|}
1757,0 \\
\end{tabular} & 3,8 & \begin{tabular}{|r|}
1776,0 \\
\end{tabular} & 3,7 & 1655,0 & 3,0 & \begin{tabular}{|r|}
990,0 \\
\end{tabular} & 1,6 & 2212,0 & 3,5 \\
\hline $\mathrm{L}$ & 33664,2 & 73,0 & 25165,8 & 53,6 & 34930,6 & 71,2 & \begin{tabular}{|l|}
34924,8 \\
\end{tabular} & 68,3 & 38918,7 & 66,33 & 37503,5 & 71,3 & 35398,0 & 68,9 & 32691,0 & 70,0 & \begin{tabular}{|l|}
34471,0 \\
\end{tabular} & 71,63 & 38937,0 & 70,1 & 44282,0 & 73,0 & 47133,0 & 73,6 \\
\hline & 46102,8 & 100,0 & \begin{tabular}{|l|l|}
46975,8 \\
\end{tabular} & 100,0 & 49088,1 & 100,0 & \begin{tabular}{|l|}
51149,81 \\
\end{tabular} & 100,0 & 58731,5 & 100,05 & 52630,8 & 100,0 & 51352,0 & 100,0 & \begin{tabular}{|l|l|}
46725,01 \\
\end{tabular} & 100,0 & \begin{tabular}{|l|}
48137,0 \\
\end{tabular} & 100,05 & 555590 & 100,0 & 6 & $100,0 \mathrm{k}$ & & 100,0 \\
\hline \multicolumn{25}{|c|}{ NAHS as a part of slowly realized assets (A3) } \\
\hline & 1666,2 & 3,6 & 1602,1 & 3,4 & 3122,1 & 6,4 & 3045,3 & 6,0 & 5702,6 & 9,7 & 2579,6 & 4,9 & 2854,0 & 5,6 & \begin{tabular}{|l|}
4799,0 \\
\end{tabular} & 10,3 & 3154,0 & 6,6 & 5169,0 & 9,3 & 6678,0 & 11,0 & 4716,0 & 7,4 \\
\hline $\mathrm{A}$ & 10884,3 & 23,6 & 10366,9 & 22,1 & 9698,8 & 19,8 & 11091,7 & 21,7 & 14075,4 & 24,01 & 14533,7 & 27,6 & 14388,0 & 28,0 & 11870,0 & 25,4 & 10604,0 & 22,01 & 12335,0 & 22,2 & 13838,0 & 22,8 & 10593,0 & 16,5 \\
\hline $\mathrm{A} 3$ & 6816,0 & 14,8 & \begin{tabular}{|l|}
5726,0 \\
\end{tabular} & 12,2 & \begin{tabular}{|l|}
6612,2 \\
\end{tabular} & 13,5 & \begin{tabular}{|l|}
6581,9 \\
\end{tabular} & 12,9 & 10354,4 & 17,6 & 8706,9 & 16,5 & 7203,0 & 14,0 & \begin{tabular}{|l|}
5085,0 \\
\end{tabular} & 10,9 & \begin{tabular}{|l|}
7017,0 \\
\end{tabular} & 14,6 & 7734,0 & 13,9 & 8408,0 & 13,9 & 14855,0 & 23,2 \\
\hline & 26736,3 & 58,0 & 29280,9 & 62,3 & 29655,0 & 60,4 & \begin{tabular}{|l|}
30430,9 \\
\end{tabular} & 59,5 & 28599,1 & 48,72 & 26810,6 & 50,9 & 26907,0 & 52,4 & 24971,0 & 53,4 & 27362,0 & 56,83 & 21,0 & 54,6 & 31740,0 & 52,3 & 33867,0 & 52,9 \\
\hline Capital & 46102,8 & 100,0 & \begin{tabular}{|l|l|}
46975,7 \\
\end{tabular} & 100,0 & 49088,1 & 100,0 & \begin{tabular}{|l|}
51149,8 \\
\end{tabular} & 100,0 & 58731,51 & 100,05 & 52630,81 & 100,0 & 51352,0 & 100,0 & 46725,01 & 100,0 & \begin{tabular}{|l|}
48137,0 \\
\end{tabular} & 100,05 & 55559,0 & 100,0 & 60664,0 & 100,0 & 64031,0 & 100,0 \\
\hline 1 & 2 & 3 & \begin{tabular}{|l|l}
4 \\
\end{tabular} & 5 & 6 & 7 & \begin{tabular}{|l|}
8 \\
\end{tabular} & 9 & 10 & 11 & 12 & 13 & 14 & 15 & 16 & \begin{tabular}{|l|l}
17 \\
\end{tabular} & 18 & \begin{tabular}{|l|}
19 \\
\end{tabular} & 20 & 21 & 22 & 23 & 24 & 25 \\
\hline & 39,2 & 0,1 & \begin{tabular}{|r|}
35,5 \\
\end{tabular} & \begin{tabular}{|l|}
0,1 \\
\end{tabular} & \begin{tabular}{|r|}
24,2 \\
\end{tabular} & 0,1 & \begin{tabular}{|r|}
23,4 \\
\end{tabular} & 0,1 & \begin{tabular}{|r|}
673,6 \\
\end{tabular} & 1,2 & 83,7 & \begin{tabular}{|l|}
0,2 \\
\end{tabular} & \begin{tabular}{|l|l|} 
& 36,0 \\
\end{tabular} & 0,07 & \begin{tabular}{|r|}
42,0 \\
\end{tabular} & 0,1 & \begin{tabular}{|r|}
162,0 \\
\end{tabular} & \begin{tabular}{|l|}
0,3 \\
\end{tabular} & 659,0 & \begin{tabular}{|r|}
1,2 \\
\end{tabular} & 290,0 & 0,5 & 473,0 & 0,7 \\
\hline & 9896,2 & 21,5 & 9626,8 & 20,5 & 11612,0 & 23,7 & \begin{tabular}{|c|}
13580,5 \\
\end{tabular} & 26,6 & 15534,8 & 26,51 & 12777,9 & 24,3 & 14123,0 & 27,5 & 12235,0 & \begin{tabular}{|l|}
26,2 \\
\end{tabular} & 11728,0 & 24,41 & 14308,0 & 25,8 & 15102,0 & 24,9 & 14213,0 & 22,2 \\
\hline & 2503,1 & 5,4 & 12147,7 & 25,9 & \begin{tabular}{|l|}
2521,3 \\
\end{tabular} & 5,1 & \begin{tabular}{|r|}
2621,1 \\
\end{tabular} & 5,1 & \begin{tabular}{|l|}
3604,3 \\
\end{tabular} & 6,1 & 2265,7 & 4,3 & \begin{tabular}{|l|l|}
1795,0 \\
\end{tabular} & 3,5 & \begin{tabular}{|l|}
1757,0 \\
\end{tabular} & 3,8 & \begin{tabular}{|l|}
1776,0 \\
\end{tabular} & 3,7 & 1655,0 & 3,0 & \begin{tabular}{|r|}
990,0 \\
\end{tabular} & 1,6 & 2212,0 & 3,5 \\
\hline & 33664,2 & 73,0 & 25165,8 & 53,6 & 34930,6 & 71,2 & \begin{tabular}{|l|}
34924,8 \\
\end{tabular} & 68,3 & 38918,7 & 66,33 & 37503,5 & 71,3 & 35398,0 & 68,9 & 32691,0 & 70,0 & \begin{tabular}{|l|}
34471,0 \\
\end{tabular} & 71,63 & 38937,0 & 70,1 & 44282,0 & 73,0 & 47133,0 & 73,6 \\
\hline & 46102,8 & 100,0 & 4607581 & 100,0 & 490881 & 100,0 & \begin{tabular}{|l|l|}
51149,8 \\
\end{tabular} & 100,0 & 58731,51 & 100,05 & 52630,81 & 100,0 & 51352,0 & 100,0 & 46725,01 & 100,0 & \begin{tabular}{|l|}
48137,0 \\
\end{tabular} & 10005 & & $1000 \mathrm{k}$ & & & 640310 & 100,0 \\
\hline \multicolumn{25}{|c|}{ NAHS as a part of fast-selling assets (A2) } \\
\hline & 666,2 & 3,6 & \begin{tabular}{|l|}
1602,1 \\
\end{tabular} & 3,4 & 3122,1 & 6,4 & 3045,3 & & 5702,6 & 9,7 & 2579,6 & 4,9 & 2854,0 & 5,6 & 4799,0 & 10,3 & 154,0 & 6,6 & 5169,0 & 9,3 & 6678,0 & 11,0 & 4716,0 & 1,7 \\
\hline $\mathrm{A} 2$ & 11072,5 & 24,0 & 10418,4 & 22,2 & 9731,0 & 19,8 & 11133,3 & 21,8 & 14103,9 & 24,01 & 14598,3 & 27,7 & 14403,0 & 28,05 & 11904,0 & 25,5 & 10701,0 & 22,21 & 12396,0 & 22,3 & 13913,0 & 22,9 & 10781,2 & 16,8 \\
\hline A3 & 6627,9 & 14,4 & 5674,5 & 12,1 & 6580,0 & 13,4 & \begin{tabular}{|l|}
6540,2 \\
\end{tabular} & 12,8 & 10325,8 & 17,6 & 8642,3 & 16,4 & 47188,0 & 14,0 & 5051,0 & 10,8 & 6920,0 & 14,4 & 7673,0 & 13,8 & 8333,0 & 13,7 & 1466,80 & 22,9 \\
\hline $\mathrm{A}$ & 26736,3 & 58,0 & 29280,9 & 62,3 & 29655,0 & 60,4 & 30430,90 & 59,5 & 28599,1 & 48,72 & 26810,6 & 50,9 & 26907,0 & 52,4 & 24971,0 & 53,42 & 27362,00 & 56,83 & 30321,0 & 54,6 & 31740,0 & 52,3 & 33867,0 & 52,9 \\
\hline & 46102,8 & 100,0 & \begin{tabular}{|l|}
46975,81 \\
\end{tabular} & 100,0 & 49088,1 & 100,0 & \begin{tabular}{|l|}
51149,8 \\
\end{tabular} & 100,0 & 58731,51 & 100,05 & 52630,81 & $100,0=$ & 51352,0 & 100,0 & \begin{tabular}{|l|l}
46725,01 \\
\end{tabular} & 100,0 & \begin{tabular}{|l|}
48137,0 \\
\end{tabular} & 100,05 & 55559,0 & 100,0 & 60664,01 & 100,0 & 64031,0 & 100,0 \\
\hline L1 & 39,2 & 0,1 & \begin{tabular}{|r|}
35,5 \\
\end{tabular} & 0,1 & \begin{tabular}{|r|}
24,2 \\
\end{tabular} & 0,1 & \begin{tabular}{|r|}
23,4 \\
\end{tabular} & 0,1 & $\begin{array}{r}673,6 \\
\end{array}$ & 1,2 & 83,7 & 0,2 & \begin{tabular}{|l|l|}
36,0 \\
\end{tabular} & 0,1 & \begin{tabular}{|r|}
42,0 \\
\end{tabular} & 0,1 & \begin{tabular}{|r|}
162,0 \\
\end{tabular} & \begin{tabular}{|l|}
0,3 \\
\end{tabular} & 659,0 & \begin{tabular}{|r|}
1,2 \\
\end{tabular} & 290,0 & 0,5 & \begin{tabular}{|l|}
473,0 \\
\end{tabular} & 0,7 \\
\hline L2 & 9896,2 & 21,5 & \begin{tabular}{|l|}
9626,8 \\
\end{tabular} & 20,5 & 11612,0 & 23,7 & \begin{tabular}{|l|}
13580,5 \\
\end{tabular} & 26,6 & 15534,8 & 26,61 & 12777,9 & 24,3 & 14123,0 & 27,5 & 12235,0 & 26,2 & \begin{tabular}{|l|}
11728,0 \\
\end{tabular} & 24,41 & 14308,0 & 25,8 & 15102,0 & 24,9 & 14213,0 & 22,2 \\
\hline & 2503,1 & 5,4 & 12147,7 & 25,9 & 2521,3 & 5,1 & 2621,1 & 5,1 & 3604,3 & 6,1 & 2265,7 & 4,3 & 1795,0 & 3,5 & \begin{tabular}{|l|}
1757,0 \\
\end{tabular} & 3,8 & 1776,0 & 3,7 & 1655,0 & 3,0 & 990,0 & 1,6 & 2212,0 & 3,5 \\
\hline $\mathrm{L} 4$ & 33664,2 & 73,0 & 25165,8 & 53,6 & 34930,6 & 71,2 & \begin{tabular}{|l|}
34924,8 \\
\end{tabular} & 68,3 & 38918,7 & 66,33 & 37503,5 & 71,3 & 35398,0 & 68,9 & 32691,0 & 70,0 & \begin{tabular}{|l|}
34471,0 \\
\end{tabular} & 71,63 & 38937,0 & 70,1 & 44282,0 & 73,0 & 47133,0 & 73,6 \\
\hline & 461028 & & & & & 100,0 & \begin{tabular}{|l|l|}
51149,8 \\
\end{tabular} & 10005 & 580 & & 526308 & & 51352,0 & 1000 & & 100,0 & 48137,0 & & & & & & & \\
\hline
\end{tabular}

Notes: A1 - absolutely liquid and highly liquid assets; A2 - fast-selling assets; A3 - slowly realized assets; A4 - hard-to-sell assets; L1 - the most urgent liabilities; L2 - current liabilities; L3 - long-term and other long-term liabilities; L4 - permanent liabilities; Capital is the total amount of the balance sheet.

Source: The authors calculations, based on the Financial Statements of PKN ORLEN (Poland) for 2007-2018

The conducted comparison of the respective asset and liabilities groups of PKN ORLEN for the years 2007-2018 allowed to determine a payment surplus or a shortage in the main relationships between asset and liability groups, in particular A1 $\geq \mathrm{L} 1, \mathrm{~A} 2 \geq \mathrm{L} 2, \mathrm{~A} 3 \geq \mathrm{L} 3, \mathrm{~A} 4 \leq \mathrm{L} 4$, the results of which are shown in Table 3. Comparative data for cases, where the NAHS is classified into different asset groups A4, A3 or A2, are shown for the purposes of identifying the impact of the NAHS on the balance sheet liquidity. This made it possible to identify differences in the amount of payment surplus or lack in each case of groupings. 


\section{ENTREPRENEURSHIP AND SUSTAINABILITY ISSUES}

ISSN 2345-0282 (online) http://jssidoi.org/jesi/ 2020 Volume 8 Number 1 (September) http://doi.org/10.9770/jesi.2020.8.1(30)

Table 3. Payment surplus (lack) of PKN ORLEN for 2007-2018 (PLN million)

(NAHS compile $0.06-0.7 \%$ of non-current assets and $0.03-0.41 \%$ of capital)

\begin{tabular}{|c|c|c|c|c|c|c|c|c|c|c|c|c|}
\hline $\mathrm{Ai}-\mathrm{Li}$ & 2007 & 2008 & 2009 & 2010 & 2011 & 2012 & 2013 & 2014 & 2015 & 2016 & 2017 & 2018 \\
\hline 1 & 2 & 3 & 4 & 5 & 6 & 7 & 8 & 9 & 10 & 11 & 12 & 13 \\
\hline \multicolumn{13}{|c|}{ NAHS as a part of hard-to-sell assets (A4) } \\
\hline A1-L1 & 1626,80 & 1566,60 & 3097,88 & 3021,97 & 5028,96 & 2495,82 & 2818,00 & 4757,00 & 2992,00 & 4510,00 & 6388,00 & 4243,00 \\
\hline A2-L2 & 988,09 & 740,12 & $-1913,20$ & $-2488,80$ & $-1459,40$ & 1755,78 & 265,00 & $-365,00$ & $-1124,00$ & $-1973,00$ & $-1264,00$ & $-3620,00$ \\
\hline A3-L3 & 4124,80 & $-6473,30$ & 4058,64 & 3919,11 & 6721,50 & 6376,64 & 5393,00 & 3294,00 & 5144,00 & 6018,00 & 7343,00 & 12454,80 \\
\hline A4-L4 & $-6739,70$ & 4166,52 & $-5243,40$ & $-4452,30$ & $-10291,00$ & $-10628,00$ & $-8476,00$ & $-7686,00$ & $-7012,00$ & $-8555,00$ & $-12467,00$ & $-13078,00$ \\
\hline \multicolumn{13}{|c|}{ NAHS as a part of slowly realized assets (A3) } \\
\hline A1-L1 & 1626,80 & 1566,60 & 3097,88 & 3021,97 & 5028,96 & 2495,82 & 2818,00 & 4757,00 & 2992,00 & 4510,00 & 6388,00 & 4243,00 \\
\hline A2-L2 & 988,09 & 740,12 & $-1913,20$ & $-2488,80$ & $-1459,40$ & 1755,78 & 265,00 & $-365,00$ & $-1124,00$ & $-1973,00$ & $-1264,00$ & $-3620,00$ \\
\hline A3-L3 & 4312,96 & $-6421,80$ & 4090,82 & 3960,76 & 6750,03 & 6441,26 & 5408,00 & 3328,00 & 5241,00 & 6079,00 & 7418,00 & 12643,00 \\
\hline A4-L4 & $-6927,80$ & 4115,03 & $-5275,50$ & $-4493,90$ & $-10320,00$ & $-10693,00$ & $-8491,00$ & $-7720,00$ & $-7109,00$ & $-8616,00$ & $-12542,00$ & $-13266,00$ \\
\hline \multicolumn{13}{|c|}{ NAHS as a part of fast-selling assets (A2) } \\
\hline A1-L1 & 1626,80 & 1566,60 & 3097,88 & 3021,97 & 5028,96 & 2495,82 & 2818,00 & 4757,00 & 2992,00 & 4510,00 & 6388,00 & 4243,00 \\
\hline A2-L2 & 1176,25 & 791,61 & $-1881,00$ & $-2447,20$ & $-1430,90$ & 1820,40 & 280,00 & $-331,00$ & $-1027,00$ & $-1912,00$ & $-1189,00$ & $-3431,80$ \\
\hline A3-L3 & 4124,80 & $-6473,30$ & 4058,64 & 3919,11 & 6721,50 & 6376,64 & 5393,00 & 3294,00 & 5144,00 & 6018,00 & 7343,00 & 12454,80 \\
\hline A4-L4 & $-6927,80$ & 4115,03 & $-5275,50$ & $-4493,90$ & $-10320,00$ & $-10693,00$ & $-8491,00$ & $-7720,00$ & $-7109,00$ & $-8616,00$ & $-12542,00$ & $-13266,0$ \\
\hline
\end{tabular}

Source: The authors calculations, based on the Financial Statements of PKN ORLEN (Poland) for 2007-2018

The main indicator of liquidity is the excess of current assets over current liabilities, and the higher this indicator is, the financial position of the company is more favourable in terms of liquidity. In general, as evidenced by the data of the Table 3, balance sheet of PKN ORLEN is relatively liquid.

The level of coverage of urgent liabilities with the most liquid assets in PKN ORLEN is sufficient [A1 $\geq$ L1], indicating that the current liquidity level of the company is adequate. Solvency in the near term is determined by the relation $[\mathrm{A} 2 \geq \mathrm{L} 2]$ and has significant limitations over the period under review, since eight years out of ten (2008-2011 and 2014-2018) have a negative balance of payments in comparing fast-selling assets and current liabilities.

An estimate of prospective liquidity at a relation of $[\mathrm{A} 3 \geq \mathrm{L} 3]$ shows, overall, the adequate solvency of PKN ORLEN in respect of long-term and other long-term liabilities, with the exception of individual years (2008), in which the coverage of long-term liabilities by slowly realized assets is impaired.

Financial stability PKN ORLEN in the long run is determined by the relation [A4 $\leq$ L4], which in most years reflects a negative balance of payments. It means that non-current assets are insufficient to cover a company's permanent liabilities. Such relation is normal and allowed in conditions of positive values of relations between assets and liabilities from previous groups.

The identified indicators of payment surplus and lack of liquid assets of PKN ORLEN testified to the disturbance of payment relations by indicators [A2-L2] in 2009-2011 and 2014-2018 and [A3-L3] in 2008. However, the ratio analysis of the company's liquidity for 2007-2018 shows a sufficient level of liquidity of its assets, despite the existing payment imbalance (Table 4). 


\section{ENTREPRENEURSHIP AND SUSTAINABILITY ISSUES}

ISSN 2345-0282 (online) http://jssidoi.org/jesi/ 2020 Volume 8 Number 1 (September) http://doi.org/10.9770/jesi.2020.8.1(30)

Table 4. Liquidity ratios of PKN ORLEN for 2007-2018

(NAHS compile $0.06-0.7 \%$ of non-current assets and $0.03-0.41 \%$ of capital)

\begin{tabular}{|c|c|c|c|c|c|c|c|c|c|}
\hline \multirow{2}{*}{ Years } & \multicolumn{3}{|c|}{ High liquidity ratio } & \multicolumn{3}{c|}{$\begin{array}{c}\text { Strict liquidity ratio } \\
\text { (acid test ratio) }\end{array}$} & \multicolumn{4}{c|}{ Quick liquidity ratio } \\
\cline { 2 - 10 } & $\begin{array}{c}\text { NAHS in } \\
\text { group A4 }\end{array}$ & $\begin{array}{l}\text { NAHS in } \\
\text { group A3 }\end{array}$ & $\begin{array}{l}\text { NAHS in } \\
\text { group A2 }\end{array}$ & $\begin{array}{c}\text { NAHS in } \\
\text { group A4 }\end{array}$ & $\begin{array}{c}\text { NAHS in } \\
\text { group A3 }\end{array}$ & $\begin{array}{c}\text { NAHS in } \\
\text { group A2 }\end{array}$ & $\begin{array}{l}\text { NAHS in } \\
\text { group A4 }\end{array}$ & $\begin{array}{c}\text { NAHS in } \\
\text { group A3 }\end{array}$ & $\begin{array}{l}\text { NAHS in } \\
\text { group A2 }\end{array}$ \\
\hline 1 & 2 & 3 & 4 & 5 & 6 & 7 & 8 & 9 & 10 \\
\hline 2007 & 0,17 & 0,17 & 0,17 & 1,26 & 1,26 & 1,28 & 1,93 & 1,95 & 1,95 \\
\hline 2008 & 0,17 & 0,17 & 0,17 & 1,24 & 1,24 & 1,24 & 1,83 & 1,83 & 1,83 \\
\hline 2009 & 0,27 & 0,27 & 0,27 & 1,10 & 1,10 & 1,10 & 1,67 & 1,67 & 1,67 \\
\hline 2010 & 0,22 & 0,22 & 0,22 & 1,04 & 1,04 & 1,04 & 1,52 & 1,52 & 1,52 \\
\hline 2011 & 0,35 & 0,35 & 0,35 & 1,22 & 1,22 & 1,22 & 1,86 & 1,86 & 1,86 \\
\hline 2012 & 0,20 & 0,20 & 0,20 & 1,33 & 1,33 & 1,34 & 2,00 & 2,01 & 2,01 \\
\hline 2013 & 0,20 & 0,20 & 0,20 & 1,22 & 1,22 & 1,22 & 1,73 & 1,73 & 1,73 \\
\hline 2014 & 0,39 & 0,39 & 0,39 & 1,36 & 1,36 & 1,36 & 1,77 & 1,77 & 1,77 \\
\hline 2015 & 0,27 & 0,27 & 0,27 & 1,16 & 1,16 & 1,17 & 1,74 & 1,75 & 1,75 \\
\hline 2016 & 0,35 & 0,35 & 0,35 & 1,17 & 1,17 & 1,17 & 1,68 & 1,69 & 1,69 \\
\hline 2017 & 0,43 & 0,43 & 0,43 & 1,33 & 1,33 & 1,34 & 1,87 & 1,88 & 1,88 \\
\hline 2018 & 0,32 & 0,32 & 0,32 & 1,04 & 1,04 & 1,06 & 2,04 & 2,05 & 2,05 \\
\hline
\end{tabular}

Source: The authors calculations, based on the Financial Statements of PKN ORLEN (Poland) for 2007-2018

According to the data of Table 4, we observe high liquidity ratio within the normal range of 0.17 to 0.43 (norm is $0.2-0.35$ ), the strict liquidity ratio (or acid test) ranges from 1.04 to 1.36 (norm to 1.0 ), the quick liquidity ratio takes on value from 1.52 to 2.05 (norm is 1.3-2.0). Considering the normative values of liquidity ratios, it can be said that PKN ORLEN during 2007-2018 has a satisfactory level of liquidity and has all the prerequisites for timely repayment of its current debt.

We performed conditional calculations based on the created models in order to identify the nature and extent of influence the operations of classifying non-current assets as held for sale on the balance sheet liquidity. These models presented the reclassification of non-current assets as NAHS in various variable volumes relative to noncurrent assets (from 5\% to 50\%) and equity PKN ORLEN (from 2\% to 30\%) during 2007-2018. The corresponding changes in the company's liquidity indicators, determined by the impact of the NAHS reclassification operations, were identified as a result (Table 5).

Table 5. Estimated effect of classification of non-current assets as held for sale on balance sheet liquidity of PKN ORLEN for 2007-2018

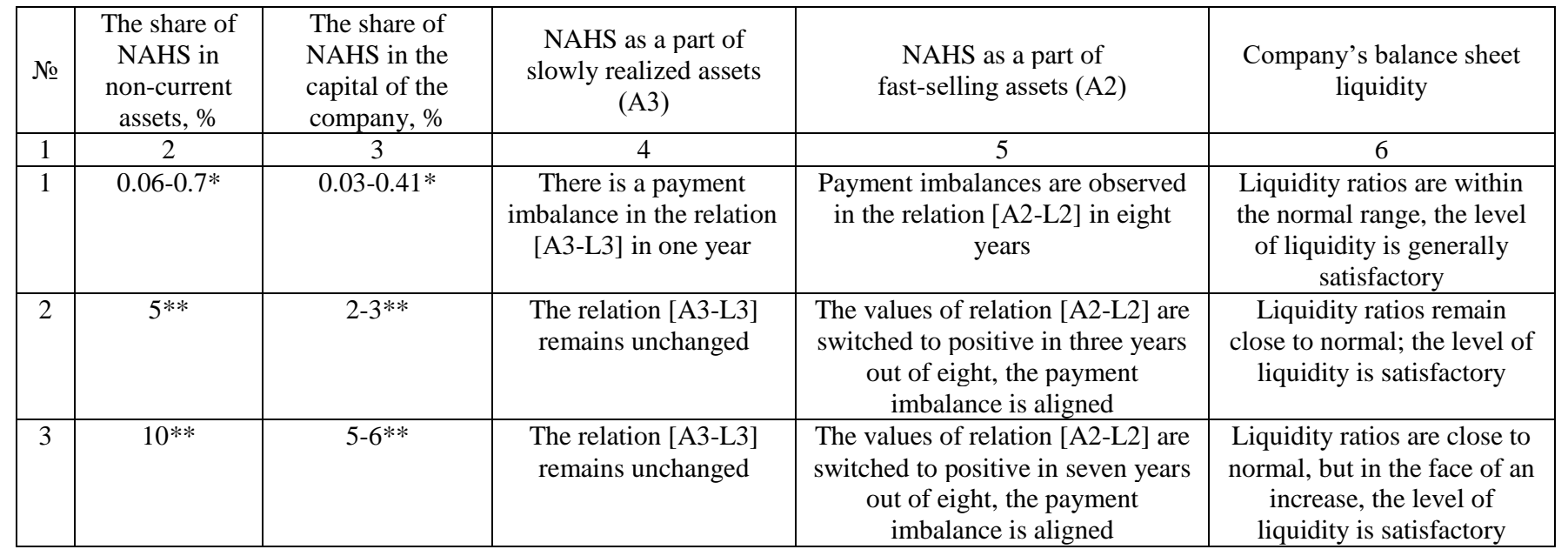




\section{ENTREPRENEURSHIP AND SUSTAINABILITY ISSUES}

ISSN 2345-0282 (online) http://jssidoi.org/jesi/ 2020 Volume 8 Number 1 (September) http://doi.org/10.9770/jesi.2020.8.1(30)

\begin{tabular}{|c|c|c|c|c|c|}
\hline 4 & $15 * *$ & $7-9 * *$ & $\begin{array}{c}\text { The relation [A3-L3] } \\
\text { remains unchanged }\end{array}$ & $\begin{array}{c}\text { The values of [A2-L2] are } \\
\text { switched to the positive in eight } \\
\text { years out of eight; the payment } \\
\text { imbalance is aligned in all the } \\
\text { studied years }\end{array}$ & $\begin{array}{c}\text { Liquidity ratios exceed the } \\
\text { norm by 0.5 points, the level } \\
\text { of liquidity becomes high }\end{array}$ \\
\hline 5 & $20 * *$ & $10-12 * *$ & $\begin{array}{c}\text { The relation [A3-L3] } \\
\text { remains unchanged }\end{array}$ & The relation [A2-L2] is positive & $\begin{array}{c}\text { Liquidity ratios exceed the } \\
\text { norm by 0.5-0.8 points, the } \\
\text { level of liquidity is high }\end{array}$ \\
\hline 6 & $30 * *$ & $15-18 * *$ & $\begin{array}{c}\text { The relation [A3-L3] } \\
\text { remains unchanged }\end{array}$ & The relation [A2-L2] is positive & $\begin{array}{c}\text { Liquidity ratios exceed the } \\
\text { norm by } 0.75-1.0 \text { points, the } \\
\text { level of liquidity is } \\
\text { excessive }\end{array}$ \\
\hline 7 & $40^{* * *}$ & $20-25 * *$ & $\begin{array}{c}\text { There is a shift in the } \\
\text { relation [A3-L3] to } \\
\text { positive, the payment } \\
\text { imbalance is aligned }\end{array}$ & The relation [A2-L2] is positive & $\begin{array}{c}\text { Liquidity ratios exceed the } \\
\text { norm by } 1-1.5 \text { points, the } \\
\text { level of liquidity is } \\
\text { excessive }\end{array}$ \\
\hline 8 & $50 * *$ & $25-30 * *$ & $\begin{array}{c}\text { The relation [A3-L3] is } \\
\text { positive }\end{array}$ & The relation [A2-L2] is positive & $\begin{array}{c}\text { Liquidity ratios exceed the } \\
\text { norm by 1.5-1.7 points, the } \\
\text { level of liquidity is } \\
\text { excessive }\end{array}$ \\
\hline
\end{tabular}

Notes: * - actual company's data; ** - author's estimates.

Source: The authors calculations, based on the Financial Statements of PKN ORLEN (Poland) for 2007-2018

The results of the calculations of the payment surplus or lack in the main relations between asset and liability groups of PKN ORLEN for the years 2007-2018 on the basis of the created models of NAHS indicators are presented in the Table 6 . This model is based on the condition that NAHS compile $40 \%$ of non-current assets and $20-25 \%$ of capital of the company. This calculation is presented due to the fact that it contains the first shift of the relation [A3-L3] from negative to positive, and has all positive changes in the relations [A2-L2].

Table 6. Payment surplus (lack) of PKN ORLEN for 2007-2018 (PLN million)

(NAHS compile $40 \%$ of non-current assets and $20-25 \%$ of capital)

\begin{tabular}{|c|c|c|c|c|c|c|c|c|c|c|c|c|c|}
\hline Ai-Li & 2007 & 2008 & 2009 & 2010 & 2011 & 2012 & 2013 & 2014 & 2015 & 2016 & 2017 & 2018 \\
\hline 1 & 2 & 3 & 4 & 5 & 6 & 7 & 8 & 9 & 10 & 11 & 12 \\
\hline \multicolumn{8}{|c|}{ NAHS as a part of hard-to-sell assets (A4) } \\
\hline A1-L1 & 1626,80 & 1566,60 & 3097,88 & 3021,97 & 5028,96 & 2495,82 & 2818,00 & 4757,00 & 2992,00 & 4510,00 & 6388,00 & 4243,00 \\
\hline A2-L2 & 988,09 & 740,12 & $-1913,20$ & $-2488,80$ & $-1459,40$ & 1755,78 & 265,00 & $-365,00$ & $-1124,00$ & $-1973,00$ & $-1264,00$ & $-3620,00$ \\
\hline A3-L3 & 4124,80 & $-6473,30$ & 4058,64 & 3919,11 & 6721,50 & 6376,64 & 5393,00 & 3294,00 & 5144,00 & 6018,00 & 7343,00 & 12454,80 \\
\hline A4-L4 & $-6739,70$ & 4166,52 & $-5243,40$ & $-4452,30$ & $-10291,00$ & $-10628,00$ & $-8476,00$ & $-7686,00$ & $-7012,00$ & $-8555,00$ & $-12467,00$ & $-13078,00$ \\
\hline \multicolumn{8}{|c|}{ NAHS as a part of slowly realized assets (A3) } \\
\hline A1-L1 & 1626,80 & 1566,60 & 3097,88 & 3021,97 & 5028,96 & 2495,82 & 2818,00 & 4757,00 & 2992,00 & 4510,00 & 6388,00 & 4243,00 \\
\hline A2-L2 & 988,09 & 740,12 & $-1913,20$ & $-2488,80$ & $-1459,40$ & 1755,78 & 265,00 & $-365,00$ & $-1124,00$ & $-1973,00$ & $-1264,00$ & $-3620,00$ \\
\hline A3-L3 & 14894,60 & $5259,69 *$ & 15933,50 & 16108,10 & 18172,60 & 17126,70 & 16161,80 & 13296,00 & 16127,60 & 18170,80 & 20069,00 & 26076,90 \\
\hline A4-L4 & $-17509,00$ & $-7566,40$ & $-17118,00$ & $-16641,00$ & $-21742,00$ & $-21378,00$ & $-19245,00$ & $-17688,00$ & $-17996,00$ & $-20708,00$ & $-25193,00$ & $-26700,00$ \\
\hline \multicolumn{8}{|c|}{ NAHS as a part of fast-selling assets (A2) } \\
\hline A1-L1 & 1626,80 & 1566,60 & 3097,88 & 3021,97 & 5028,96 & 2495,82 & 2818,00 & 4757,00 & 2992,00 & 4510,00 & 6388,00 & 4243,00 \\
\hline A2-L2 & 11757,90 & 12473,10 & $9961,71 *$ & $9700,17 *$ & $9991,64 *$ & 12505,90 & 11033,80 & $9637,00 *$ & $9859,60 *$ & $10179,80 *$ & $11462,00 *$ & $10002,10 *$ \\
\hline A3-L3 & 4124,80 & $-6473,30$ & 4058,64 & 3919,11 & 6721,50 & 6376,64 & 5393,00 & 3294,00 & 5144,00 & 6018,00 & 7343,00 & 12454,80 \\
\hline A4-L4 & $-17509,00$ & $-7566,40$ & $-17118,00$ & $-16641,00$ & $-21742,00$ & $-21378,00$ & $-19245,00$ & $-17688,00$ & $-17996,00$ & $-20708,00$ & $-25193,00$ & $-26700,00$ \\
\hline
\end{tabular}

Note: *Indicators in which the relation [A3-L3] shifted to the positive due to increased volumes of NAHS to 20-25\% of the company's capital. The switching of the relations [A2-L2] to the positive began to occur with increasing of NAHS volumes to 2-3\% of the company's capital.

Source: The authors calculations, based on the Financial Statements of PKN ORLEN (Poland) for 2007-2018 


\section{ENTREPRENEURSHIP AND SUSTAINABILITY ISSUES}

ISSN 2345-0282 (online) http://jssidoi.org/jesi/ 2020 Volume 8 Number 1 (September) http://doi.org/10.9770/jesi.2020.8.1(30)

The data in Table 6 indicates existing changes in the balance of payments of PKN ORLEN in 2008, 2009-2011 and 2014-2018 as a result of changes in the asset structure and classification of non-current assets as hold for sale in the amount of $40 \%$ of non-current assets and $20-25 \%$ of the company's capital. Accordingly, the liquidity ratios of the balance sheet also changed (Table 7).

Table 7. Liquidity ratios of PKN ORLEN for 2007-2018

(NAHS compile $40 \%$ of non-current assets and $20-25 \%$ of capital)

\begin{tabular}{|c|c|c|c|c|c|c|c|c|c|}
\hline \multirow{2}{*}{ Years } & \multicolumn{3}{|c|}{ High liquidity ratio } & \multicolumn{3}{c|}{$\begin{array}{c}\text { Strict liquidity ratio } \\
\text { (acid test ratio) }\end{array}$} & \multicolumn{4}{c|}{ Quick liquidity ratio } \\
\cline { 2 - 10 } & $\begin{array}{c}\text { NAHS in } \\
\text { group A4 }\end{array}$ & $\begin{array}{l}\text { NAHS in } \\
\text { group A3 }\end{array}$ & $\begin{array}{l}\text { NAHS in } \\
\text { group A2 }\end{array}$ & $\begin{array}{c}\text { NAHS in } \\
\text { group A4 }\end{array}$ & $\begin{array}{l}\text { NAHS in } \\
\text { group A3 }\end{array}$ & $\begin{array}{l}\text { NAHS in } \\
\text { group A2 }\end{array}$ & $\begin{array}{l}\text { NAHS in } \\
\text { group A4 }\end{array}$ & $\begin{array}{c}\text { NAHS in } \\
\text { group A3 }\end{array}$ & $\begin{array}{l}\text { NAHS in } \\
\text { group A2 }\end{array}$ \\
\hline 1 & 2 & 3 & 4 & 5 & 6 & 7 & 8 & 9 & 10 \\
\hline 2007 & 0,17 & 0,17 & 0,17 & 1,26 & 1,26 & 2,35 & 1,93 & 3,01 & 3,01 \\
\hline 2008 & 0,17 & 0,17 & 0,17 & 1,24 & 1,24 & 2,45 & 1,83 & 3,04 & 3,04 \\
\hline 2009 & 0,27 & 0,27 & 0,27 & 1,10 & 1,10 & 2,12 & 1,67 & 2,69 & 2,69 \\
\hline 2010 & 0,22 & 0,22 & 0,22 & 1,04 & 1,04 & 1,94 & 1,52 & 2,42 & 2,42 \\
\hline 2011 & 0,35 & 0,35 & 0,35 & 1,22 & 1,22 & 1,93 & 1,86 & 2,56 & 2,56 \\
\hline 2012 & 0,20 & 0,20 & 0,20 & 1,33 & 1,33 & 2,17 & 2,00 & 2,84 & 2,84 \\
\hline 2013 & 0,20 & 0,20 & 0,20 & 1,22 & 1,22 & 1,98 & 1,73 & 2,49 & 2,49 \\
\hline 2014 & 0,39 & 0,39 & 0,39 & 1,36 & 1,36 & 2,17 & 1,77 & 2,58 & 2,58 \\
\hline 2015 & 0,27 & 0,27 & 0,27 & 1,16 & 1,16 & 2,08 & 1,74 & 2,66 & 2,66 \\
\hline 2016 & 0,35 & 0,35 & 0,35 & 1,17 & 1,17 & 1,98 & 1,68 & 2,49 & 2,49 \\
\hline 2017 & 0,43 & 0,43 & 0,43 & 1,33 & 1,33 & 2,16 & 1,87 & 2,70 & 2,70 \\
\hline 2018 & 0,32 & 0,32 & 0,32 & 1,04 & 1,04 & 1,97 & 2,04 & 2,97 & 2,97 \\
\hline
\end{tabular}

Source: The authors calculations, based on the Financial Statements of PKN ORLEN (Poland) for 2007-2018

As can be seen from the Table 7, the level of liquidity of the balance sheet of PKN ORLEN has changed significantly as a result of changes in the asset structure and exceeds the normative values by an average of 1-1.5 points. It should be noted here that the estimated liquidity level, given in Table. 7 for the company, is excessive, especially considering its scope of activities, such as the oil and gas industry, which is capital intensive and requires significant amounts of machinery and equipment to ensure normal operations. However, the calculations made it possible to identify critical changes in the volume of non-current assets held for sale, which require the analysts' attention when conducting financial statements analysis and stakeholders when making decisions based on such analysis.

\section{Conclusions}

The conducted analysis of liquidity of the oil and gas company PKN ORLEN (Poland) for 2007-2018 showed how its liquidity indicators and balance of payments by groups of assets and liabilities change, under the influence of classification of non-current assets as held for sale. The calculations are made taking into account the inclusion of NAHS into the different groups of assets by the degree of their liquidity, in particular A4, A3 та A2.

Conducted liquidity analysis of PKN ORLEN, based on its 2007-2018 actual data, showed that overall the company's balance sheet is comparatively liquid but has some warning indicators. In particular, the company has an adequate level of current liquidity, and its current liabilities are sufficiently covered by the most liquid assets. Solvency in the near term has significant limitations, whereas a negative balance of payments is observed eight years out of ten (2008-2011 and 2014-2018) and fast-selling assets do not cover current liabilities. The prospective liquidity assessment generally showed that the company has an adequate level of solvency in respect of long-term and other long-term liabilities, except for individual years (2008), in which long-term liabilities are 


\section{ENTREPRENEURSHIP AND SUSTAINABILITY ISSUES}

ISSN 2345-0282 (online) http://jssidoi.org/jesi/

2020 Volume 8 Number 1 (September)

http://doi.org/10.9770/jesi.2020.8.1(30)

not covered by slowly realized assets. Long-term financial sustainability is normal taking into account the proper level of current coverage of the company's liabilities.

Despite the existing payment imbalances, the company's liquidity analysis for 2007-2018 showed a sufficient level of liquidity of its assets. The calculated ratios of high liquidity, strict liquidity (or acid test) and quick liquidity are within the recommended normative values, and the company has all the prerequisites for timely repayment of its current debt.

Conditional calculations were performed based on the created models to identify the nature and extent of the impact of the classification of non-current assets as held for sale on the balance sheet liquidity of PKN ORLEN. These models reflect the result of the reclassification of non-current assets in the NAHS in different variable volumes relative to non-current assets (from 5\% to 50\%) and capital (from 2\% to 30\%) during 2007-2018. As a result, relevant changes of the company's liquidity ratios identified by impact of the NAHS classification operations.

According to the analysis, taking into account the inclusion of NAHS in different groups of assets by their degree of liquidity (A4, A3 and A2), the switching the values of relation [A2-L2] to positive starts when the non-current assets held for sale compile 5\% and more of non-current assets and 2-3\% of the company's capital. There is also an impact on the balance sheet liquidity that begin to gain in excess (exceeding the norm by 0.5 points and more) when non-current assets held for sale compile $15 \%$ and more of non-current assets and $7-9 \%$ of the value of capital.

For the purposes of liquidity analysis in the grouping of assets according to their degree of liquidity, NAHS can be included in both the A3 group of slowly realized assets and the A2 group of fast-selling assets. A reasonable choice of group A3 or A2 should be made taking into account the available conditions of sale for each object of NAHS separately. The period during which the asset will be sold should be performed as the basic indicator for grouping. In particular, if the sales plan of NAHS ensures their alienation within 6 months, this is a reliable basis for qualifying assets as fast-selling assets (A2). If, however, the NAHS sale is foreseen for a period from 6 months to a year, then the NAHS should be assigned to a slowly realized asset group (A3).

In order to determine the optimum indicator of NAHS in a company's balance sheet, which allows to avoid the risk of diminishing productivity of available production capacities and at the same time contribute to the release of financial resources as a result of the sale of assets, it should be taken into account the fact that switching the relation [A2-L2] to positive begins to occur when non-current assets held for sale compile from 5\% and more of non-current assets and 2-3\% of the value of capital. Balance sheet liquidity ratios should also be considered as they begin to gain in excess when the non-current assets held for sale compile $15 \%$ and more of non-current assets and $7-9 \%$ and more of the value of capital.

Accordingly, the optimal indicator of NAHS in the balance sheet of the company is the volume of 5 to $10 \%$, which allows to align the balance of payments without the risk of reducing productivity. NAHS values up to 5\% will not have a significant impact on the liquidity of the balance sheet; values greater than $15 \%$ can have a critical impact on the overall performance of non-current assets.

Restrictions on the application of the above methodology for analysing the impact of classification of non-current assets as held-for-sale on the balance sheet liquidity of the company may be short-term sales transactions of NAHS that do not reflect the value of NAHS in the interim or annual financial statements of the company. In such cases, analysts have to provide a special request for information to the company in terms of the value of NAHS, the time period of their retention in the accounts and sales transactions. Also, to accurately assess the liquidity and solvency of the company, cash flow data during the reporting period should be taken into account. 


\section{ENTREPRENEURSHIP AND SUSTAINABILITY ISSUES}

ISSN 2345-0282 (online) http://jssidoi.org/jesi/

2020 Volume 8 Number 1 (September)

http://doi.org/10.9770/jesi.2020.8.1(30)

\section{References}

Cherep, A., Puhal'ska, A. 2011. Analysis of the availability and efficiency of the use of intangible assets by the machine-building enterprises of the Zaporizhzhya region. International collection of scientific works, 2(19), 468-477.

Chub, Y. 2014. Analysis of the availability and use of intangible assets. Economic analysis: a collection of scientific works, Ternopil National Economic University, 15(3), 225-229.

Cieśla, A. 2016 December 27. Rozliczenie sprzedaży środka trwałego (Settlement of fixed asset sales). Gazeta Podatkowa, 103(1353). Retrieved from https://www.gofin.pl/podatki/17,2,61,161094,rozliczenie-sprzedazy-srodka-trwalego.html

Gabrusewicz, W. 2018. Analiza struktury aktywów (majątku) (Analysis of the structure of assets). Analiza-prognoza finansowa. Niezbędnik analityka. Retrieved from https://analizy-prognozy.pl/analizy-prognozy-finansowe/analiza/wskaznikowa/struktury/majatku/

International Financial Reporting Standard 5 Non-current Assets Held for Sale and Discontinued Operations. Retrieved from https://library.croneri.co.uk/cch_uk/iast/ifrs5-200403

Ivashevska, O.M., 2006. Accounting and Analysis of Liquid Assets: Abstract. of PhD Thesis in Economics. Kyiv, 22 p.

Kalubi, R., 2017. Impact of inflation on capital assets analysis. International scientific and research journal, 3(57), Ch. 2, 74-80.

Khalatur, S.M., 2016. Analysis of efficiency of use of non-current assets of agricultural enterprise, IV International scientific conference "Formation of effective model of enterprise development in the conditions of market economy" November 24-25, Zhytomyr: ZhSTU. 233235.

Kraszewska-Szuba, M., 2017. Jak księgować i rozliczyć sprzedaż środka trwałego (How to book and settle sales of a fixed asset), Rachunkowość. Retrieved from http://www.rp.pl/Rachunkowosc/312139978-Jak-ksiegowac-i-rozliczyc-sprzedaz-srodka-trwalego.html

Kul'ko-Labintseva, I., 2014. Analysis of the structure of fixed assets of «Motor Sich» PC. Country and Regions. Economics and Entrepreneurship, 2(77), 102-105.

Lazarowicz, A., 2018. Sprzedaż środka trwałego a wycofanie majątku na cele prywatne (Sale of a fixed asset and withdrawal of assets for private purposes). Poradnik Przedsiębiorcy. Retrieved from https://poradnikprzedsiebiorcy.pl/-dokumentowanie-sprzedazy-srodkowtrwalych.

Nawaiseh, M.E., 2015. Impairment Analysis of Non-current Assets under DCF Based-test in the Jordanian Industrial Shareholding Companies. British Journal of Economics, Management \& Trade, 7(1), 10-22.

Paliukh, M., Matviychuk, L., 2015. Assessment of the condition and use of fixed assets in the agrarian sector of the economy. Sustainable Development of Economy, 2, 208-214.

PKN ORLEN / Website. Annual Reports for 2007-2018. Retrieved from https://www.orlen.pl/EN/InvestorRelations/AnnualReport/Pages/default.aspx

Polyova, T., Dubovets, M., 2010. Analysis of the efficiency of the use of intangible assets through the example of «Savinsky Sugar Plant» PC. Economic strategy and prospects of trade and services industries development, 1, 91-96.

Rozheliuk, V., Denchuk, P., 2006. System of intangible assets`condition and use indicators, Scientific notes: a collection of scientific works of the Department of Economic Analysis of the Ternopil National Economic University, Ternopil, 15, 49-51.

Selivanova, N., Tkachenko, Y., Popko, Y., 2016. Features of the methods for conducting analysis of fixed assets of the enterprise, Materials of the VII International scientific Internet conference "Problems of the market and development of regions of Ukraine in the XXI century», December 5-9, Odessa: Odessa National Polytechnic University, 98-102.

Stupnytska, T.M., 2014. Influence of structural changes of Form "Balance sheet" on analysis of enterprise liquidity. Food Industry Economics, 3(23), 75-79. 


\section{ENTREPRENEURSHIP AND SUSTAINABILITY ISSUES}

ISSN 2345-0282 (online) http://jssidoi.org/jesi/ 2020 Volume 8 Number 1 (September)

http://doi.org/10.9770/jesi.2020.8.1(30)

Teren, G.M., 2016. Enterprise Liquidity Analysis Metric. Investment: Practice and Experience, 8, 36-41.

Tyuleneva, N.O., 2013. Modern Methods of Balance Sheet Liquidity Analysis, Accounting and Finance Problems, 4(12), 61-65.

Mariya M. SHYGUN is Doctor of Science in Economics (Accounting, analysis and audit), Professor, Certified Auditor of Ukraine and Chair of the Accounting and Taxation Department at the Kyiv National Economic University named after Vadym Hetman (Kyiv, Ukraine). She is an author of more than 200 publications, including 10 monographies as a writer and co-writer, study books, scientific articles in journals indexed in international scientometric databases. Leader and participant in more than 12 research on accounting, auditing and taxation. Awarded for active participation in the development of audit in Ukraine, as a recognized expert in this field by the "Honor of the Audit Chamber of Ukraine" (2016). Research interests: theory and methodology of accounting and auditing; modeling of accounting system; strategic managerial accounting; systemic connections between management, accounting and control.

ORCID ID: https://orcid.org/0000-0003-1660-9534

Natalia A. OSTAPIUK - Doctor of Economics (Econ.), Professor, Department of Accounting and Taxation, Kiev National Economics University named after Vadym Hetman, Certified Auditor, Certified Specialist in IFRS. Work experience in the bodies of the State Treasury of Ukraine, audit firms, training centers for teaching accounting for accountants, business owners, law enforcement officials. Research interests: problems of implementation and application of IFRS and IPSAS, taxation and control practice.

ORCID ID: $\underline{\text { https://orcid.org/0000-0003-2347-3815 }}$

Oksana V. ZAIACHKIVSKA: PhD (economic sciences), Associate Professor of the Department of Finance and Environmental Economics at the National University of Water and Environmental Engineering, Ukraine, Rivne. Research interests: financial security, pricing

ORCID ID: https://orcid.org/0000-0002-8792-9204

Natalya V. GOYLO - Ph.D. (Econ.), Associate professor of the audit department of Kyiv National Economic University named after Vadym Hetman. Experience as an accountant in a holding company, assistant auditor. Took part in the development of software for reporting under IFRS and related disclosures in XBRL format for the banking and insurance sectors of the Ukrainian economy. Research interests: automation of accounting and audit; internal audit and internal control system.

ORCID ID: $\underline{\text { https://orcid.org/0000-0002-5526-7322 }}$

Make your research more visible, join the Twitter account of ENTREPRENEURSHIP AND SUSTAINABILITY ISSUES:

@Entrepr69728810

Copyright (C) 2020 by author(s) and VsI Entrepreneurship and Sustainability Center

This work is licensed under the Creative Commons Attribution International License (CC BY).

http://creativecommons.org/licenses/by/4.0/

(c) (i) Open Access 